\title{
Best Efforts to Track Relation Using Data Mining "VincaRelation Tracker"
}

\author{
Venkatesh M Badiger ${ }^{1}$, Nilam V Divekar ${ }^{2}$, Monali D Zende ${ }^{3}$ \\ ${ }^{1,2,3}$ Vincalabs Infotech Pvt. Ltd., Pune (Maharashtra), India
}

\begin{abstract}
VincaRelationTracker system is about tracking relations in all categories. This system is to provide relation details or information of a person or individual in various categories they are related to such as community, organization, friends, family and many more. In this system the data is secure that is been provided by the person and that data is not shared to any third party or anyone.
\end{abstract}

Keywords: Direct and Indirect Relation, Relation Tractor, Relation Order, Family Hierarchy, Gender Ratio, Population

\section{Introduction}

In day to day life, everyone maintains birthday information of relatives, friends and closed ones in mobiles or systems, but no one maintains complete family information neither all friends circle nor all closed ones information. Even though they maintain some information, its temporary storage as mobiles or system can be at fault. Also, if anyone maintains all information, the information is only in one order i.e. direct and opposite relation information is not achieved. Eg. $\mathrm{A}$ is brother of $\mathrm{B}$, but $\mathrm{B}$ is brother/sister of $\mathrm{A}$ is not maintained. Searching data of particular member and his relatives is almost impossible. It's is also very difficult to track and keep details of every relation of different categories. It is hard to keep track of the person date of birth and their demise date. Also, relatives bank details no one knows, which is very important aspect in terms of monetary after his / her demise.

To overcome all real life scenarios's, we have brought up a helping hand project "VincaRelationTracker" for Indian population and their relations. "VincaRelationTracker" system will be very useful in future point of view because with this we can get the relation of a particular person in every field he is related to like friends, family, organization, community etc.

We can keep track of it and this system give us hierarchy structure of the relation that person is related to.

The user can see his all relation any time anywhere as it is an online application.

User interacts with large amount of data and fetches the data from large amount of data called as data mining. The interesting patterns are represented to the user, and may be stored as new data in the database. According to this view, In entire process Data mining is only one step, an essential one since it uncovers hidden patterns for evaluation.

Data Mining is also called as knowledge discovery process. In "VincaRelationTracker" System user fetch the relations from database. Therefore in this paper we use the term data mining. We adopt a broad view of data mining functionality: data mining is the process of fetching interesting pattern from large amounts of data stored either in databases, data warehouses, or other information repositories.

Data mining is the process of sorting large data sets to identify patterns and establish relationships to solve problems through data analysis. Data mining tools allow enterprises to predict future trends.

Data mining, the extraction of hidden predictive information from large databases, is a powerful new technology with great potential to help companies focus on the most important information in their data warehouses.

Data mining is a process used by companies to turn raw data into useful information. By using software to look for patterns in large batches of data, businesses can learn more about their customers and develop more effective marketing strategies as well as increase sales and decrease costs.

\section{System Architecture}

"VincaRelationTracker" System detects the all categories relationships. "VincaRelationTracker" system is useful to the individual as he can get all relations he is related to in various categories anytime anywhere in a well structured format. It makes the system user-friendly. It enforces security measures to avoid unauthorized access to customer records.

In Figure1 For registration on the system the user has to contact the Manager he has to submit his details to the Manager. Manager then registers the user after registration the user get a confirmation message on his email -id. The Manager then gives them credentials (username and password) after his registration).The user than can login into the system to see his relations related to him if he has any queries related to his own data or he want to update his information then he has to contact the Manager for that purpose. If the user forgets his password he has to enter his registered email-id after that he will get the recovery password through which he can login into the System.

When User wants their relationship data he can get their relationship data in tree structure format.

In System architecture Vinca-Supervisor creating the Sub- 


\section{International Journal of Science and Research (IJSR) \\ ISSN (Online): 2319-7064}

Index Copernicus Value (2016): 79.57 | Impact Factor (2015): 6.391

Supervisor, Sub-Supervisor creating Manager and VincaSupervisor assigning the task to the manager and manager handles the user.

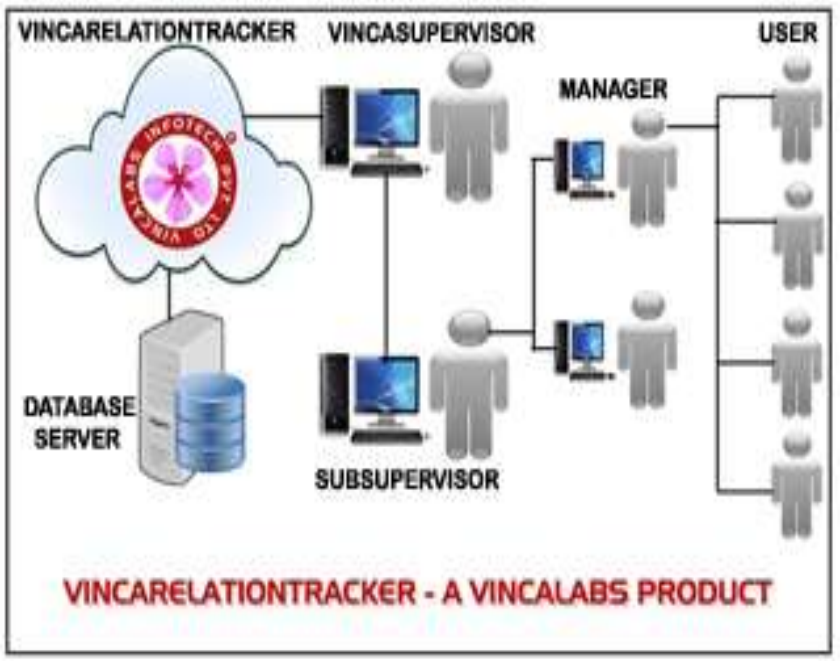

Figure 1: System Architecture

\section{Equations}

Our purpose is to get relation from second person to first person based on opposite gender.

The inputs provided are first person and second person. The Inputs also contain gender of these persons. The input also maintains relationship from first person to second person.

$$
\begin{aligned}
& A l \leftrightarrow \operatorname{Arr} \\
& \operatorname{Al}(n) \leftrightarrow \operatorname{Arr}(n)
\end{aligned}
$$

$\forall n=1: 5$ Where Al(1)is First Person

Where Al(2)is Gender First Person

Where Al(3)is Direct Relations

Where Al(4)is Second Person

Where Al(5)is Gender of Second Person

$$
\begin{aligned}
& \text { RelAl1 } \leftrightarrow \text { Reln }[1] \\
& \operatorname{RelAl} 2 \leftrightarrow \operatorname{Reln}[2] \\
& \operatorname{RelAl} 3 \leftrightarrow \operatorname{Reln}[3] \\
& \operatorname{RelAl} 4 \leftrightarrow \operatorname{Reln}[4] \\
& \text { RelAl5 } \leftrightarrow \text { Reln }[5]
\end{aligned}
$$

From Equation (1)

Consider $A l 3(i)$

$$
\text { If RelAl1 }[i]=A l 3[i]
$$

Display Result as RelAl2[i]

Where $i$ is from 1 to $n, n$ is total number of records

\section{Conclusion}

In this "VincaRelationTracker" system, we are maintaining direct relations of family members along with their important dates such as birthdate, demise dates, etc. Using this system, we retrieve opposite relations based on gender. Also, we use data mining concepts for filtering techniques. Our future scope is to maintain blood groups, gender ratio, unique surnames.

\section{References}

[1] www.vincalabs.com "For Complete Relationship Status"

[2] www.vincalabs.com/vrt "For Relationship Methods VincaRelationTracker jar using "

[3] Morgan Kaufman "Data Mining Concepts and Techniques, $3^{\text {rd }}$ Edition"

[4] Prof Dr .Karsten Borgwardt“ Data Mining :ReferencesETH Zurich"

[5] K. Deb, S. Agrawal, A. Pratab, T. Meyarivan, "A Fast $\mathrm{J}$.

\section{Author Profile}

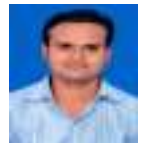

Mr. Venkatesh M. Badiger received the B.Sc.(Computer Science) and M.Sc.(Computer Science) degrees from University of Pune in 2007 and 2009, respectively. He has been certified as Computer Hacking Forensic Investigator and IBM RAD. He is working as $\mathrm{CEO}$ and Director at Vincalabs Infotech Pvt. Ltd. He is also working as Independent Social Worker with Police Official \& Legal Administration for Cyber Security.

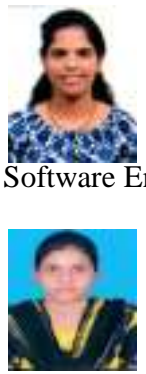

Ms. Nilam V. Divekar received the B.E.(Computer Engineering) and M.E.(Computer Engineering) degrees from University of Pune in 2013 and 2016 respectively. She is currently associated as Associate Engineer.

Ms. Monali D. Zende received the B.E.(Computer Engineering) and M.E.(Computer Engineering) degrees from University of Pune in 2014 and 2016 respectively. She is currently associated with Vincalabs Infotech Pvt. Ltd. as Associate Software Engineer. 